\title{
ON THE TIDAL FUNCTION BETWEEN TWO REAL BODIES
}

\author{
V. SHKODROV, V. IVANOVA \\ Institute of Astronomy, \\ Bulgarian Academy of Sciences \\ 1784 Sofia, Blvd. Tsarigradsko shosse, 72
}

\begin{abstract}
The general expression of the tidal function between two real bodies of arbitrary mass distribution has been derived. The case has been regarded when the distances between the bodies are comparable to their sizes. In this case, the effect of the body figure is substantial.

The relations obtained are utilized for the tidal influence of Jupiter on the surface of Io. It has been shown that Jupiter's shape (with $J_{2}=$ 0.015 ) causes a deformation in the sub-Jupiter point of about $15 \mathrm{~m}$. This is approximately $10 \%$ of the total tidal deformation of Io caused by Jupiter's gravitation.
\end{abstract}

\section{General theory}

When the tidal effect of a celestial body A on another celestial body B is determined, the first body is usually treated as a mass point $A$. This is so because the distances between the bodies are large and because the difference between the real figure of the tide formation body and the spherical symmetry in its mass distribution is negligibly small. In this case, the tidal function of the body $\mathrm{A}$ on the body $\mathrm{B}$ is given by the usual expression

$$
W_{0}=\frac{4}{3} G(r) \sum_{n=2}\left(\frac{\rho}{r}\right)^{n-2} P_{n}(\cos H),
$$

where $G(r)$ is the known Dutson constant [1]

$$
G(r)=\frac{4}{3} \frac{f M_{A}}{r}\left(\frac{\rho}{r}\right)^{2},
$$




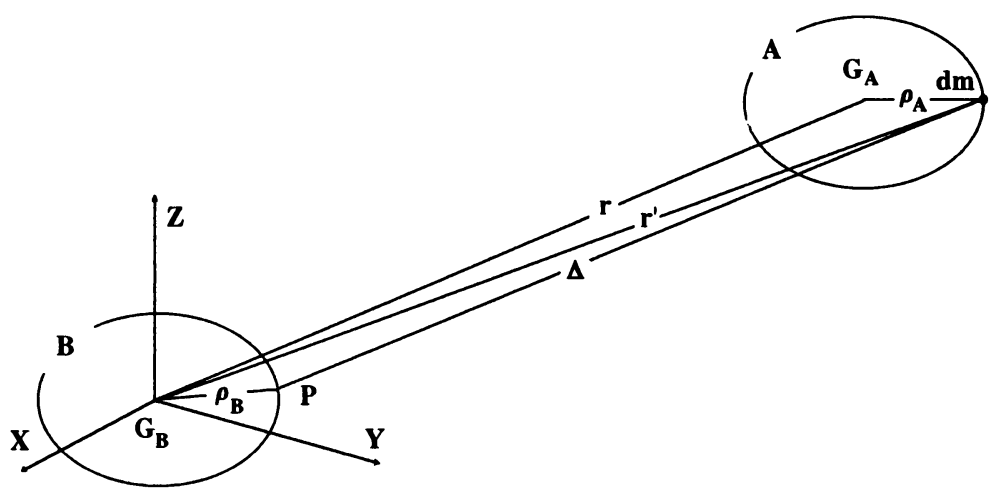

$\mathbf{r}$ is the position vector of the point where the mass $M_{A}$ is located, and $P n(\cos H)$ is the Legendre polynomial with an argument $\cos (H)=\mathbf{r}_{0} \rho_{0}$, $\left(\mathbf{r}_{0}=\mathbf{r} / r ; \rho_{0}=\rho / \rho\right)$.

In this paper, we analyze the case when the distance $R$ between the bodies $A$ and $B$ is not great and our purpose is to find the tidal influence of the real body $\mathrm{A}$ on $\mathbf{B}$ (Fig. 1). Here, the tidal function $W$ at point $\mathbf{P}$ is determined by

$$
W=V_{p}-V_{G_{B}}=f M_{A} \int\left(\frac{1}{\Delta}-\frac{1}{r^{\prime}}\right) \frac{d m}{M_{A}},
$$

where $M_{A}$ is the mass of the body $\mathrm{A}, d m$ is an elementary mass; $r^{\prime}$ and $\Delta$ are the distances from $d m$ to the mass center $G_{B}$ and to the point $\mathrm{P}$, respectively (Fig. 1). If $\Delta^{-1}$ is expressed by volume spherical functions [2], then

$$
\Delta^{-1}=\sum_{n=0, m} \frac{1}{2 n+1} T_{n}^{m *}\left(\rho_{p}\right) N_{n}^{m}\left(\mathbf{r}^{\prime}\right)
$$

where

$$
\begin{gathered}
T_{n}^{m *}\left(\rho_{p}\right)=\rho_{p}^{n} Y_{n}^{m *}(\tau), \\
N_{n}^{m}\left(\mathbf{r}^{\prime}\right)=\frac{1}{r^{n+1}} Y_{n}^{m}\left(\tau^{\prime}\right) .
\end{gathered}
$$

In (4), $Y_{n}^{m *}(\tau)$ and $Y_{n}^{m}\left(\tau^{\prime}\right)$ are surface spherical functions whose arguments are the components of the unit vectors $\tau$ and $\tau^{\prime}$ in a spherical coordinate system with an origin $\mathrm{G}$. By $\left({ }^{*}\right)$ we denote the intricate conjugated quantity to $Y_{n}^{m}(\tau)$.

Hence, for $V_{p}$, we obtain

$$
V_{p}=f M_{A} \sum_{n=0, m} \frac{1}{2 n+1} T_{n}^{m *}\left(\boldsymbol{\rho}_{p}\right) \int_{T_{A}} N_{n}^{m}\left(\mathbf{r}^{\prime}\right) \frac{d m}{M_{A}} .
$$


and, from (1), (4) and (5),

$$
W=f M_{A} \sum_{n=2, m} \frac{1}{2 n+1} T_{n}^{m *}\left(\rho_{p}\right) \int_{T_{A}} N_{n}^{m}\left(\mathbf{r}^{\prime}\right) \frac{d m}{M_{A}} .
$$

The last expression is obtained by taking into account that $N_{0}^{0}\left(\mathbf{r}^{\prime}\right)=$ $-1 / r^{\prime}$ and that the harmonic with an index $\mathrm{n}=1$ does not cause a tidal effect.

To connect the tidal function $W$ with the harmonic coefficients characterizing the distribution of mass in $T_{A}$, we shall refer $N_{n}^{m}\left(\mathbf{r}^{\prime}\right)$ to the origin $G_{A}$ of $T_{A}$. For this purpose, we use the relation (Fig. 1)

$$
\mathbf{r}^{\prime}=\mathbf{r}+\rho_{A},
$$

and $N_{n}^{m}\left(\mathbf{r}+\boldsymbol{\rho}_{A}\right)$ is presented according to the rule described in [2] in the following form:

$$
\Omega N_{n}^{m}\left(\mathbf{r}+\rho_{A}\right)=\sum_{(L, M)} Q_{L M n m} T_{n}^{m *}\left(\boldsymbol{\rho}_{A}\right) N_{n+L}^{m+M}\left(\mathbf{r}^{\prime}\right) .
$$

In (8), $\Omega$ denotes the Taylor operator whose action is identical to the translation of the origin of the coordinate system by $G_{B}$ at $G_{A}$, while

$$
Q_{L M n m}=\frac{(-1)^{L}[(2 n+1)(n-m+L-M) !(n+m+L+M) !]^{1 / 2}}{[(2 L+1)(2 n+2 L+1)(n+m) !(n-m) !(L+M) !(L-M) !]^{1 / 2}} .
$$

The action of the operator $W$ on $N_{n}^{m}\left(\mathbf{r}^{\prime}\right) \in G_{B}$ leads to the appearance of

$$
T_{M *}^{L}\left(\boldsymbol{\rho}_{A}\right) \in G_{A} ; N_{n+L}^{m+M}\left(\mathbf{r}^{\prime}\right) \in G_{B} .
$$

The final expression for the tidal function $W$ of the real body $\mathrm{A}$ on $\mathrm{B}$ is obtained from (5) and (8) in the form

$$
W=f M_{A} \sum_{n=2, m, L, M} Q_{L M n m} \frac{2 L+1}{2 n+1} A_{L}^{M} T_{n}^{m *}\left(\rho_{p}\right) N_{n+L}^{m+M}(\mathbf{r})
$$

where

$$
A_{L}^{M}=\frac{1}{2 L+1} \int_{T_{A}} T_{L}^{M *}\left(\rho_{A}\right) \frac{d m}{M_{A}}
$$

are the harmonic coefficients characterizing the mass distribution in the body $T_{A}$.

Expression (10) generalizes the usual presentation of the tidal potential where the body causing the tides is treated either as a point mass $\mathrm{M}$ or as 
a spherical body with spherical symmetry of its mass distribution. Really, if in (10) the term with $L=M=0$ is separated, we obtain the expression

$$
W=f M_{A} \sum_{n=2, m} \frac{1}{2 n+1} T_{n}^{m *}\left(\boldsymbol{\rho}_{p}\right) N_{n}^{m}(\mathbf{r})+W_{q},
$$

the first part of which is, in fact, the usual presentation (1) of the tidal potential, while the second part may be presented in the following form

$$
\begin{gathered}
W_{q}=\frac{4}{3} G(r) \sum_{n m L M} Q_{L M n m} \frac{2 L+1}{2 n+1}\left(\frac{\rho_{p}}{R}\right)^{n-2}\left(\frac{R}{r}\right)^{n+L-2} \\
. A_{L}^{M *} Y_{n}^{m *}\left(\theta_{p}, \lambda_{p}\right) Y_{n+L}^{m+M}(\theta, \lambda),
\end{gathered}
$$

where $R$ is constant, $q, l$ and $q_{p}, l_{p}$ are the spherical coordinates of the directions $\mathbf{r}$ and $\mathbf{r}_{p}$, respectively, and

$$
A_{L}^{M *}=\frac{1}{2 n+1} \int_{T_{B}}\left(\frac{\rho_{A}}{R}\right)^{L} Y_{L}^{M *}\left(\theta_{A}, \lambda_{A}\right) \frac{d m}{M_{A}} .
$$

The summing is performed according to the scheme

$$
\sum_{(n, m, L, M)}=\sum_{(n=2, m=-n)}^{n} \sum_{(L=2, M=-L)}^{L}
$$

The relation (12) gives the tidal effect related to the mass distribution of the body.

\section{The tidal function of Jupiter}

The relations obtained are utilized for the tidal influence of Jupiter on the surface of Io. It has been shown that Jupiter's shape (with $J_{2}=0.015$ ) causes a deformation in the sub-Jupiter point of about $15 \mathrm{~m}$. This is approximately $10 \%$ of the total tidal deformation of Io caused by Jupiter's gravitation.

The investigations made for this paper were thanks to the financial support by National Foundation "Scientific Investigations" under contract Ph. 477/94, with Ministry of Education, Science and Technology.

\section{References}

1. Melchior P. (1971), Physique et dynamique, Planetaires, Vol.1

2. Shkodrov V. (1989), Planet Potential, Publishing House of Bulgarian Academy of Sciences, Sofia. 